\title{
PRELIMINARY RESULTS ON BEAM DYNAMICS OF LASER PULSE SHAPING EFFECTS IN SPARC
}

\author{
M. Boscolo, M. Ferrario, M. Migliorati, INFN- Frascati, Italy \\ F. Castelli, S. Cialdi, A. Flacco, INFN-Milano, Italy
}

\begin{abstract}
Electron beams with different initial pulse characteristics have been transported along the SPARC photoinjector. The differences we considered come from the possible profiles of the laser pulse driving the radiofrequency (rf) electron gun. Realistic deviations from the ideal flat-top pulse give for example a ramp or multipeaks shape with a relative rise time, plateau deformation and roughness. The beam quality of the electron source is essential for the production of high brightness beams. The role played by the laser pulse is crucial, as it determines the electron beam pulse shape.

The study presented here gives some indications on the tolerances of the laser beam characteristics for the electron beam quality preservation. The different beam current profiles are transported along the injector with the PARMELA [1] code. The electron pulse as external input to PARMELA is generated directly from a laser pulse resulting from certain configurations of the SPARC laser system. The variations of normalized emittance (ENX) and envelope are discussed for the different cases. Spectral and shaping requirements on the laser pulse temporal profile for the SPARC injector are found.
\end{abstract}

\section{INTRODUCTION}

High brilliance rf electron guns are driven by laser pulses of rectangular profile [2]. However, the shape of the laser pulse is very sensitive to the setting of the pulse shaping system as well as to the alignment of the many optical elements of the laser system [3,4]. The final output waveform comes out different from the expected target pulse, as alignment and position of the components are perturbed with respect to the ideal setting. The profile deviations due to possible system perturbations have been detected with computer simulations. We assume no pulse deviation from cathode emitting surface, so that the emitted electron beam profile matches the laser profile. This assumption is fairly good for a metallic photocathode.

Because of the very small chance of having the ideal output laser pulse it is worth investigating the beam dynamics versus the different possible pulse characteristics. We take into considerations in our discussion the rise time, the amplitude and frequency modulation in the plateau and a possible ramp shape of the pulse. We stress that thanks to the shaping system the pulse characteristics can be controlled separately.

Their impact on the beam evolution along the linac has been investigated using the PARMELA code with a sample of 15000 particles. The FWHM of the generated pulses have been fixed to $10 \mathrm{ps}$, in order to compare results with the nominal case. The SPARC accelerator has been optimized for minimizing both the total projected emittance and the slice emittance, knowing that the slice value is critical for the SASE process. However, a good matching of the beam along the accelerator is only possible if the total projected emittance is quite small.

We point out that the tool we have developed allows performing complete start-to-end simulations starting from the generation of the laser pulse up to the FEL/SASE studies through the undulator. We think this tool will result very useful during the tests of the different laser profiles and during the machine commissioning with real pulses.

\section{EMITTANCE VERSUS ELECTRON PULSE CHARACTERISTICS}

We present in the following the results of the emittance for some selected different cases.

\section{Emittance Versus Rise Time}

The best profile of an electron pulse in relation to the goal of emittance minimization leads to two relative emittance minima which have the same value [5]. This is obtained by a rectangular pulse with zero rise and decay time (defined as the time interval from $10 \%$ to $90 \%$ of the total pulse height). The longer the rise time the higher the value of the first minimum and, in turn, the higher the final emittance. This is, in fact, shown by the simulations depicted in fig.1.

The emittance increase in the end of the beam line versus the rise time, calculated for the last working conditions, reproduces substantially other investigations [6].

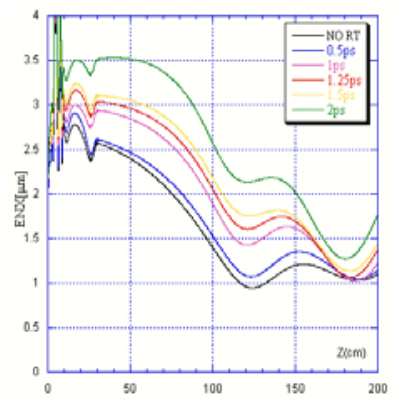

Figure 1: Beam emittance evolution along the first $200 \mathrm{~cm}$ of the SPARC injector without accelerating structures for different rise times.

For completeness, we have checked the behaviour with $1 \mathrm{ps}$ rise time and 2 ps decay time, and vice versa. The final emittance is near equal to the one obtained with both rise and decay time selected to 2 ps. This result can be 
interpreted by saying that space-charge forces tend to homogenize the phase-space occupied by the beam. The longitudinal phase spaces is very similar for the two cases, therefore there is negligible influence on the emittance.

The simulation results indicate clearly the strict requirement on the rise time. A 1 ps of rise time is a really a challenging goal for a laser system, as pointed out also in [7].

\section{Emittance Versus Plateau Fluctuations}

The profile of a pulse generated by a laser will have, in general, amplitude and frequency modulations. First effects have been investigated in [6]. Now we have separated the effects on the emittance by the two types of modulations: an amplitude modulation and a frequency modulation, an example of them are represented in fig. 2(a), and in fig. 2(b). Rise and decay times are fixed at the nominal value of $1 \mathrm{ps}$. (a)

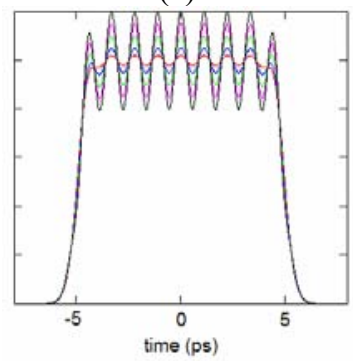

(b)

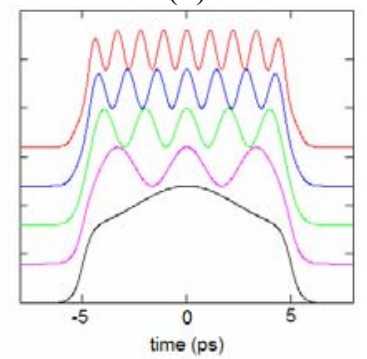

Figure 2: Frame (a): Intensity temporal profiles with different amplitude oscillations in the flat-top for a fixed frequency of $0.9 \mathrm{ps}^{-1}$. Frame (b): Intensity temporal profiles with different oscillation frequencies on the plateau for a fixed amplitude of $20 \%$.

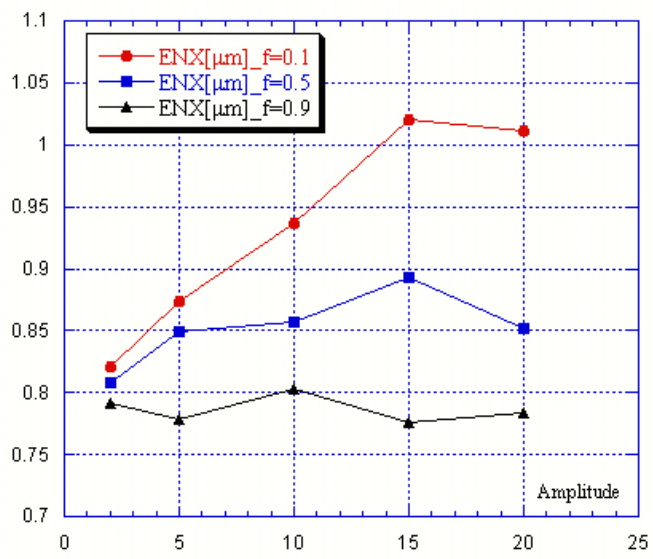

Figure 3: Projected horizontal emittance at the end of the linac versus the amplitude oscillations for frequencies of $0.1 \mathrm{ps}^{-1}$ (red dots), $0.5 \mathrm{ps}^{-1}$ (blue squares) and $0.9 \mathrm{ps}^{-1}$ (black triangles).

The effect of the amplitude modulation is analyzed assuming frequencies of $0.9 \mathrm{ps}^{-1}, 0.5 \mathrm{ps}^{-1}$ and $0.1 \mathrm{ps}^{-1}$. The result of the investigations is shown in fig. 3 . The value of the emittance remains substantially constant increasing the amplitude of the oscillations, as long as the frequency is sufficiently high. The slope of the emittance-amplitude line decreases with the increase of the frequency.

A possible interpretation of this behavior is that a cylinder of charge with a high frequency density modulation is a dense array of charged disks. When the distance between the disks is short enough the electric field nearby the border is very similar to that of a uniform charge cylinder. This could be the reason of the equal action by the applied magnetic field on both charge distributions. In addition, we note that the longitudinal density oscillations of the electron distribution disappear after a drift of $150 \mathrm{~cm}$, in the meanwhile an energy oscillation shows up, as discussed in [6] and [8].

\section{EMITTANCE VERSUS A PULSE HAVING A RAMP PROFILE}

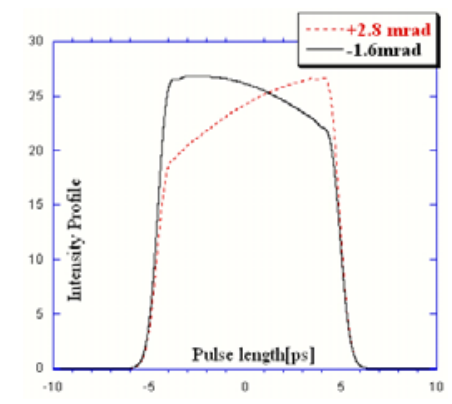

Figure 4: Intensity profiles assume a ramp shape for different values of crystal tilting angles.

The temporal profile of the laser pulse at $266 \mathrm{~nm}$ is required to be of rectangular fashion with fast rise time. A rectangular profile is generated inserting in a laser system a shaping device (shaper) which transforms the Gaussianlike profile generated by a laser oscillator into the target one. Moreover the propagation through the crystals generating the second and the third harmonics can produce distortions on the spatial and temporal profiles, and the shaper system must provide an active setting of the optical components to control the profile deformations, as discussed in [9].

In general light pulse manipulation is obtained by amplitude and phase modulation of its spectral components. One of the proposed pulse shaper systems can be constituted by a Dazzler located before the amplifier, and a stretcher after, or before, the crystals for the third harmonic generation. The Dazzler introduces an amplitude modulation on the spectrum and a small chirp in order to obtain a pulse of a few ps.

We now study the case in which a rectangular laser pulse, after a stretching to the requested 10ps length, crosses a harmonic generating crystal assuming a ramp fashion, due to a mismatch on the matching angle between the light propagation direction and the principal axis of the birefringent crystal. We note that a perfect alignment is a really difficult achievement. We have 
considered the misalignment angles of $\pm 2.8 \mathrm{mrad}$ and $\pm 1.6 \mathrm{mrad}$. Negative angles mean higher leading edge of the pulse profile (see Fig. 4).

The simulation has shown that a negative ramp leads to emittance enhancement whereas a positive ramp does not influence the emittance, as shown in Fig. 5.

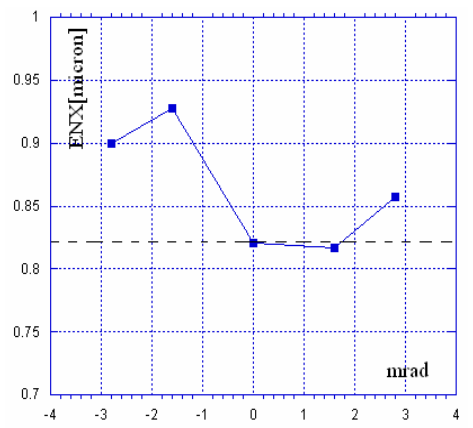

Figure 5: The projected emittance at the end of the linac versus crystal tilting angle. The dashed line is the reference emittance value for a perfectly aligned crystal.

\section{A REALISTIC PULSE}

As a final investigation we have done the simulation for the laser pulse of Fig. 6 .

This laser pulse is obtained setting the laser system on the basis of the trade off between the two requirements of high conversion efficiency and good rectangular pulse profile, analyzed in [9]. Furthermore, it is assumed that one of the two up-converting crystals has a misalignment angle of $1.6 \mathrm{mrad}$.

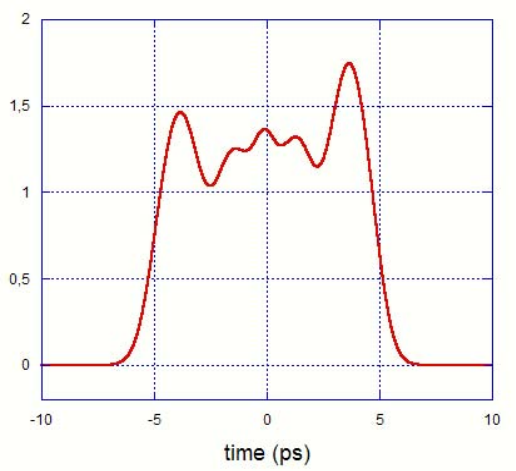

Figure 6: Profile of a realistic pulse obtained with a typical configuration of a laser system.

The objective of efficient harmonic conversion implies a high intensity of the entering pulse. Because of this, the Gaussian initial pulse was stretched only up to 2 ps before the up-conversion. The rest of the stretching is applied after the harmonic generation to the final $10 \mathrm{ps}$ temporal length. This procedure of pulse forming leads to a reasonably good conversion efficiency but at the cost of an increase in the rise time to about $1.6 \mathrm{ps}$. For these choices the final electron beam results with the three peaks and with a ramp fashion.

The simulation result with this pulse gives an emittance of $\mathrm{ENX}=0.85 \mu \mathrm{m}$, that is nearly equal to the one obtained with the rectangular pulse of $10 \mathrm{ps}$ with $1.5 \mathrm{ps}$ of rise time. Apparently, the only relevant parameter for the emittance preservation seems the rise time in this particular simulation. This result needs further investigations.

We mention that out code that generates the electron bunch does not take into consideration the effect that the quantum efficiency (QE) depends on frequency. Forthcoming studies will include this effect in the modelling.

\section{CONCLUSIONS}

The detailed study of the effects on the emittance of the different characteristics of the laser/electron pulse gives the relevance of each characteristic in relation to the emittance degradation. The emittance comes out to be sensible firstly to the rise time and secondly to small frequencies and smoothed modulations on the plateau. Fast bumps on the flat top do not provide detectable emittance variations. A weak increase of the emittance comes out when the pulse has a negative ramp shape, whereas it remains unchanged with opposite ramp.

Since the large and complex laser systems which drive an rf-gun have inherently perturbation of the setting of the many elements, and since a good rectangular pulse profile is very sensitive to perturbations, it is advisable to set the laser system in a feedback loop configuration because a proper shaping system has the adaptive capability to recover the distortions introduced by the perturbations.

\section{ACKNOWLEDGMENTS}

The work is partly supported by Ministero Istruzione Università Ricerca, Progetti Strategici, DD 1834, Dec.4,2002 and European Contract RII3-CTPHI506395CARE.

\section{REFERENCES}

[1] J.Billen, "PARMELA", LA-UR-96-1835, 1996.

[2] M. Biagini et al., 'SPARC photoinjector working point optimization, tolerances and sensitivity to errors', Proc. of EPAC04 Lucerne, July 2004.

[3] S. Cialdi and I.Boscolo, 'A laser pulse shaper for the lowemittance radiofrequency SPARC electron gun', Nucl. Instr. And Meth., A 526 (2004) p.239-248.

[4] S. Cialdi and I.Boscolo, A shaper for providing long laser target waveforms', Nucl. Instr. and Meth., A 538 (2005) p.1-7.

[5] M. Ferrario et al., "Homdyn study for the LCLS Photoinjector", SLAC-PUB-8400, Mar 2000.

[6] M. Biagini et al, "Beam Dynamics Studies for the SPARC Project”, Proc. of PAC03, p. 2077, 2003.

[7] H. Loos et al., "Temporal e-beam shaping in an S-Band Accelerator", this Conference.

[8] C. Limborg at al., "Sensitivity studies for the LCLS photoinjector beamline", Nucl. Instr. and Meth., A 528 (2004) p.350-354.

[9] S.Cialdi, F.Castelli and I.Boscolo, "Rectangular pulse formation in a laser harmonic generation", INFN/BE05/01; S.Cialdi, F.Castelli and I.Boscolo, submitted to J.Opt.Soc.Am. B. 